Revue Revue de l'histoire des religions

$$
\begin{array}{cl}
\text { de I histoire } & \begin{array}{c}
3 \mid 2012 \\
\text { des religions }
\end{array} \\
\text { Varia }
\end{array}
$$

\title{
Alexandre GRANDAZZI, Alba Longa. Histoire d'une légende
}

Rome, École française de Rome («Bibliothèque des Écoles Françaises d'Athènes et de Rome ", 336), 2008, XV-988 p., 25 cm,

ISBN 978-2-7283-0412-7.

Jean-Claude Richard

\section{OpenEdition}

Journals

Édition électronique

URL : http://journals.openedition.org/rhr/7916

DOI : $10.4000 /$ rhr.7916

ISSN : 2105-2573

Éditeur

Armand Colin

Édition imprimée

Date de publication : 1 septembre 2012

Pagination : 416-418

ISBN : 978-2200-92975-0

ISSN : 0035-1423

Référence électronique

Jean-Claude Richard, "Alexandre grandazzI, Alba Longa. Histoire d'une légende », Revue de l'histoire des religions [En ligne], 3 | 2012, mis en ligne le 04 octobre 2012, consulté le 22 septembre 2020. URL : http://journals.openedition.org/rhr/7916 ; DOI : https://doi.org/10.4000/rhr.7916

Ce document a été généré automatiquement le 22 septembre 2020.

Tous droits réservés 


\section{Alexandre GRANDAZZI, Alba Longa. Histoire d'une légende}

Rome, École française de Rome (« Bibliothèque des Écoles Françaises d'Athènes et de Rome ", 336), 2008, XV-988 p., 25 cm, ISBN 978-2-7283-0412-7.

Jean-Claude Richard

\section{RÉFÉRENCE}

Alexandre GRANDAZZI, Alba Longa. Histoire d'une légende. Rome, École française de Rome ("Bibliothèque des Écoles Françaises d'Athènes et de Rome », 336), 2008, XV-988 p., $25 \mathrm{~cm}$, ISBN 978-2-7283-0412-7.

1 Cette thèse est le couronnement d'une série d'études dont la première, publiée en 1986, ouvrait la voie que l'auteur a depuis systématiquement défrichée, puis élargie. Dès cette date, il avançait de sérieuses raisons de douter qu'Albe ait jamais été une «ville ». Si les témoignages anciens affirment le contraire, c'est que la pseudo-histoire des primordia avait été conditionnée en ce sens par la célébration des Féries Latines qui avait lieu chaque année sur le mons Albanus. Pour ses représentants, un rite de cette ampleur appelait au départ un cadre urbain. Le livre de Grandazzi fera date. En effet, la légende d'Albe y est étudiée "dans toute sa portée et toutes ses composantes ». À preuve ses trois parties dont la première est consacrée au cadre spatio-temporel (relief, réseau hydrographique et climat) du paysage albain, la seconde aux données archéologiques et la troisième aux Feriae Latinae ainsi qu'à la tradition des rois d'Albe. Ajoutons qu'au catalogue exhaustif des découvertes faites dans la zone des monts Albains qui complète celui de P.E. Gierow, la deuxième partie juxtapose une discussion approfondie de la vexatissima quaestio de la localisation d'Albe : elle est conduite à la lumière du débat encore récent sur le "proto-urbain ", puisque certains archéologues cherchent à sauver l'honneur en substituant, dans leur approche du problème, la notion de réalité "proto-urbaine» à celle de ville. On saura gré à l'auteur d'avoir regroupé, avant 
d'entrer in medias res, tous les témoignages anciens qui parlent d'Albe comme d'une ville. La même volonté de présenter un dossier complet des citations antiques apparaît dans les chapitres respectivement consacrés, l'un aux Féries Latines, l'autre aux rois d'Albe.

2 De la première partie, nous dirons seulement que l'auteur a su tirer profit d'acquis récents de diverses sciences de la nature, parmi lesquelles la sismologie et la vulcanologie à propos de la crue prodigieuse du lac d'Albe. Parmi les realia Albana, qui sont au cœur de la deuxième partie, le développement intitulé « Les vivants et la terre » mérite de retenir l'attention, à plus d'un titre. Quant au chapitre VIII intitulé «Les errances d'une ville fantôme ", il règle le problème. Le témoignage de l'archéologie est en effet décisif: le nombre des centres albains et leurs faibles dimensions « excluent toute forme d'une entité [...] d'une nature urbaine ». Les arguments invoqués par les tenants de la théorie traditionnelle sont des leurres ou relèvent de l'héroïsme superflu. D'où la conclusion qui s'impose : ce sont l'importance et le caractère solennel des Féries Latines qui firent accéder à l'être une ville qui n'avait jamais existé. Pour la date de formation de cette légende, l'auteur propose soit le $\mathrm{IV}^{\mathrm{e}}$ siècle, soit le début du $\mathrm{V}^{\mathrm{e}}$ voire le $\mathrm{VI}^{\mathrm{e}}$. Ses préférences vont à la première. Les nôtres aussi.

Intitulé Sacra Albana, le chapitre IX étudie tous les aspects (localisation, date - qui varie puisque ce rituel appartient au groupe des fêtes mobiles -, liturgie) de cette solennité. L'analyse suit, mais en le remontant, l'ordre chronologique, démarche permettant de mieux appréhender les permanences et les évolutions. Les Féries Romaines sont bien sûr antérieures à - 451. Certaines de nos sources en attribuent la fondation à l'un des Tarquins. En soulignant qu'il ne s'agissait pas d'une création ex nihilo, l'auteur penche à juste titre pour cette doctrine et note que la dualité des réunions tenues sous le dernier règne au caput Ferentinae et du rituel du Latiar ne saurait infirmer cette chronologie. Les premières traduisaient l'emprise que Rome exerçait désormais sur la Ligue Latine. Les Feriae l'étendaient à la solennité albaine et répondaient à des préoccupations tenant à la qualité religieuse d'un sommet sur lequel se dressait peut-être un auguraculum faisant pendant à celui dont l'existence est attestée à Rome sur le collis Latiaris... La présence à ce rituel d'un consul au moins inviterait à supposer qu'il s'y rendait auspicandi causa. Cette prise d'auspices aurait inauguré son imperium sur un territoire qui avait longtemps symbolisé pour la Ville le monde extérieur. Mais si ce raisonnement se fonde sur des correspondances déjà utilisées par d'autres, la réalité d'une prise d'auspices sur le mont Albain nécessaire à la plénitude de l'imperium n'est pas, tant s'en faut, établie et ne laisse pas de faire problème à nos yeux. L'auteur en a si clairement conscience qu'il s'en tient sur ce point à des généralités prudentes. En effet, rien de comparable ne semble attesté à propos du sacrifice que les plus hauts magistrats romains accomplissaient à Lavinium au début de leur charge plus probablement qu'à sa fin.

Quant aux Féries Latiales déjà centrées sur le culte rendu par les Latins à Jupiter Latial, elles associaient dans leur célébration les communautés regroupées dans une ligue placée sous son patronage. La liste des 30 populi Albenses qui nous a été conservée par Pline l'Ancien garde le souvenir d'une réalité "préurbaine, préromaine et préétrusque ». Ce document était "authentique, ancien et complet » en même temps que "non exhaustif » parce que "périégétique » (cf. les considérations subtiles des p. 720-721 qui ne règlent pourtant pas tous les problèmes). Pline l'avait sans doute emprunté à Varron, lui-même tributaire de Caton qui aurait transcrit une inscription 
albaine encore visible à son époque. Il pouvait en théorie dater du moment où, dans les années - 450, Rome commença à graver les fastes des Féries Latines. Mais il remontait plus probablement à la réorganisation par l'un des Tarquins, au cours $\mathrm{du} \mathrm{VI}^{\mathrm{e}}$ siècle, d'un rituel plus ancien alors annexé par l'Urbs. Dans la proportion d'un sur trois, les noms regroupés dans cette liste sont entachés d'incertitude. Un ensemble de simulations bien conduites permet à Grandazzi de mettre en évidence des regroupements géographiques de populi, donc de sites constituant autant de forteresses naturelles. À ses yeux, cette liste reflète une situation qui porte la marque des $\mathrm{X}^{\mathrm{e}}$-VIII ${ }^{\mathrm{e}}$ siècles.

Que, dans le principe, elle garde le souvenir d'un état de choses fort ancien est à nos yeux une quasi-certitude. Le point de vue de l'auteur se fonde en dernière analyse sur la présence en son sein de noms qui semblent être ceux de groupes fixés sur le site de ce qui n'était pas encore Rome. Il n'en serait pas moins étonnant, selon nous, que quelques populi associés aux tout premiers temps du Latiar n'aient pas disparu au cours d'une durée comprise entre deux et quatre siècles. «Primordial » dans son principe, le document peut néanmoins porter la marque d'« aggiornamenti ». S'il a conscience de cet état de choses, A. Grandazzi ne s'arrête pas sur ce problème qu'il évacue en deux phrases.

6 L'autre pièce majeure du dossier concernant Albe venu jusqu'à nous est la liste de ses rois, d'Ascagne à Numitor. On considère en général que, destinée à combler l'écart entre l'arrivée d'Enée dans le Latium vers - 1200 et la fondation de Rome, elle est postérieure aux recherches chronologiques d'Eratosthène. L'historicité des rois en question fait l'objet d'un rejet quasi général auquel l'auteur souscrit. L'étude qu'il nous propose de ce document innove sur des points majeurs. Enfin, stricto sensu, les rois d'Albe restèrent reges Albanorum.

7 L'examen des noms qui garnissent les diverses listes autorise un certain nombre de conclusions. Plusieurs, ceux de Silvius, Latinus, Picus, Faunus nous reportent à un contexte latial ; d'autres (Tiberinus, Aventinus, Agrippa, Aremulus, Amulius et Numitor et peut-être Procas) constituent la composante romaine de ce document. Enfin, particulièrement significative aux yeux de l'auteur, l'inclusion de Capys parmi les rois d'Albe remonte vraisemblablement aux premières décennies de l'état romanocampanien qui naquit en - 340. On le mettra en parallèle avec certaines traditions qui faisaient honneur à Rhômos d'avoir fondé Capoue. Bref, au terme de développements alertes et contre la communis opinio, A. Grandazzi rend un verdict d'authenticité sur la légende des rois d'Albe qu'il définit comme "très ancienne dans son principe, assez uniforme et précocement stabilisée » (p. 885). Reste qu'à nos yeux, par les glissements qu'elle autorise, l'assimilation liste des rois/légende royale ne va peut-être pas sans risque s'il est vrai que chacune de ces catégories appelle une approche différente.

Cela dit, cette thèse est une grande thèse, par l'ampleur de la documentation mise en œuvre et des perspectives qu'elle ouvre. L'auteur sait où il va et son lecteur n'a pas de peine à le suivre. Écrit avec un rare bonheur d'expression où le sens de la formule le dispute à l'habileté, ce travail magistral est lourd d'acquis, mais aussi de promesses. Dans un premier temps, il faut espérer que, sur sa lancée, A. Grandazzi nous donne la mise au point qui s'impose sur le problème des gentes albanae. 


\section{AUTEURS}

JEAN-CLAUDE RICHARD

Université Paris IV - Sorbonne. 